\title{
THE LEVEL OF IgG ANTIBODIES REACTIVE TO TF, Tn AND ALPHA- GaI POLYACRYLAMIDE-GLYCOCONJUGATES IN BREAST CANCER PATIENTS: RELATION TO SURVIVAL
}

\author{
E.P. Smorodin*, B.L. Sergeyev \\ Department of Oncology and Immunology, National Institute for Health Development, \\ Hiiu 42, Tallinn 11619, Estonia
}

\begin{abstract}
Background: The serum levels of IgG antibodies reactive to glycoconjugates (TF, Tn and $\alpha \mathrm{Gal}$ ) were found to be associated with prognosis of gastrointestinal cancer patients. Aim: To study the relation between the levels of serum antibodies to TF-pAA, Tn-PAA and $\alpha$ Gal-PAA polyacrylamide-based glycoconjugates and survival in breast cancer. Materials and Methods: The preoperative level of IgG antibodies was analysed in the serum of patients $(n=59)$ using ELISA with polyacrylamide-glycoconjugates namely, TF-pAA (amide-type), and ethanolamide-conjugates Tn-PAA and $\alpha$ Gal-PAA. Survival rate and hazard ratio (HR) were assessed by the Kaplan - Meier method and Cox univariate analysis in different pathomorphological groups. Results: Significantly better survival was observed in patients with an increased level of anti-TF-pAA antibodies both for all patients in total and groups in stages II-III; N1-2 and G3 $(p=0.008-0.021, \mathrm{HR}=0.18-0.23$, mean survival time in months 164-186 vs 69-121). A trend to worse survival was observed in increased level of anti-Tn IgG (stages II-III) and anti- $\alpha$ Gal IgG (G3): $p=0.075, \mathrm{HR}=2.49$ and $p=0.066, \mathrm{HR}=3.27$, respectively. Conclusion: The method for the determination of circulating anti-TF-pAA IgG may be a useful supplement in long-term prognostic assessment of patients with breast cancer.
\end{abstract}

Key Words: breast cancer, survival, glycoconjugates, antibodies, microbiota, dysbiosis.

\section{INTRODUCTION}

Resident microbiota in humans colonizes boundary surfaces maintaining immune homeostasis [1]. Imbalance in microbial communities, or microbial dysbiosis, has been shown to contribute to the cancerous pathogenesis and progression including breast cancer [2, 3]. Microbial glycans are key antigens in host-microbial interactions [4]. Blood group related cryptic glycans Thomsen - Friedenreich (TF, Galß1-3GalNAca), Tn (GalNAca) and aGal (Gala1-3Galß) and/or mimicking determinants are present in enteric bacteria [5-7]. Besides, TF and Tn are tumor-associated glycans (TAG) because they are frequently expressed in adenocarcinomas. The increased expression of TF and Tn is associated with the tumor invasion and metastases [8-10]. The aGal epitope is aberrantly expressed in human cells and it may be involved in autoimmune diseases [7]. Being a constant source of foreign antigens, microbiota stimulates the production of anti-glycan antibodies. It is considered that the natural anti-TF, -Tn and - $a$ Gal antibodies in human are induced by enteric microorganisms $[5,7]$. The high IgG antibody level and its change may reflect the adaptive immune response of the host to microorganisms or increased autoreactivity to self antigens. Comparative data about spontaneously occurring anti-glycan antibodies in cancer, their autoreactivity to "self" and "altered self" antigens including TAG deserve a circumstantial investigation. We demonstrated

Submitted: April 05, 2016.

${ }^{\star}$ Correspondence: $\quad$ Tel.: +372-6593934; fax: +372-6593901;

E-mail: jevgeni.smorodin@tai.ee

Abbreviations used: HR - hazard ratio; IEF - isoelectric focusing; PAA - polyacrylamide; $R$ - responders; WR - weak responders; TAG - tumor-associated glycans; TF - Thomsen - Friedenreich glycan; Tn - GalNAca; aGal - (Gala1-3Galß). the prognostic significance of the level of serum IgG antibodies to some glycans, and its relation to clinical parameters, tumor progression and histopathological grading in gastrointestinal cancer patients [11-13]. The antibody response to microbial glycans and its relation to survival has not been characterized in patients with breast cancer yet.

The aim of the present study was to evaluate whether the level of IgG antibodies to TF-pAA, Tn-PAA and aGal-PAA is related to survival of patients with breast cancer, taking also into consideration the stage of the disease, tumor volume, morphology and the tumor spread in regional lymph nodes.

\section{MATERIALS AND METHODS}

Patients. The investigation was carried out in accordance with the ICH GCP Standards and approved by Tallinn Medical Research Ethics Committee, Estonia. The informed consent was obtained from each patient under examination. Females with breast cancer in stages I-III $(n=59)$ whose diagnosis was verified by the pTNM system [14], were included in the study. The chemo- and X-ray therapy-prescribed patients as well as patients who died of causes other than tumor recurrence were excluded from the study. The median and mean age \pm SD was 51.5 and $52.1 \pm 9.4$.

Glycoconjugates. The synthetic glycoconjugates that are homogenous antigens with a single reiterative glycotope [15] were obtained from Lectinity Holding Inc. The soluble TF conjugate with $\mathrm{N}$-unsubstituted polyacrylamide (TF-pAA, amide-type) as well as ethanolamide-type glycoconjugates of the poly[N-(2hydroxyethyl)acrylamide] namely, Tn-PAA and aGalPAA $\left(B_{\mathrm{di}}-\mathrm{PAA}\right)$ were used in ELISA. The TF-pAA contained $0.1 \mathrm{~mol}$ of TF per $1 \mathrm{~mol}$ of pAA. The rest of the conjugates had $0.2 \mathrm{~mol}$ of a saccharide per $1 \mathrm{~mol}$ 
of PAA. Tris(hydroxymethyl)aminomethane-PAA was used as an adequate negative control [16].

Indirect ELISA. The assay was performed as described earlier [11]. The sera were kept at $4{ }^{\circ} \mathrm{C}$ for no longer than three weeks or were frozen $\left(-50{ }^{\circ} \mathrm{C}\right)$ and thawed once before use. The IgG antibody level was estimated as a ratio of $A_{\text {test }} / A_{\text {control }}$, where $A_{\text {test }}$ is the absorbance with the glycoconjugate and $A_{\text {control, }}$ with the Tris-PAA. The variation coefficient was $3 \%$.

Isoelectric focusing (IEF) and Western blotting. IEF was performed in a 1\% agarose IEF (GE Healthcare Bio-Sciences AB, Sweden) containing 10\% glycerol as described in [17]. A pH gradient was formed in the gel with 4\% Ampholytes high resolution 3-10 (SigmaAldrich, USA) and 1\% Pharmalyte 5-8 (GE Healthcare Bio-Sciences AB, Sweden).

Statistical analysis. The levels of antibodies were studied in relation to overall survival time starting from the date of the preoperatively taken blood sample. The Kaplan - Meier method was used for the estimation of survival curves in the univariate analysis of patients groups, the significance of differences was analyzed by the log-rank test. The Cox proportional hazards model was used to evaluate the risk of death. Statistical analysis was performed using SPSS, version 22.0. The median (M) of the level of anti-TF-pAA, -Tn and -aGal antibodies was used as cut-off to discriminate between responders ( $R$, the level above or equal to $M$ ) and weak responders (WR, the level below M). Survival of R vs WR was evaluated in subgroups divided by stages, tumor volume, tumor grade and regional lymph node metastases. The difference in survival between patient groups was considered to be significant when $p \leqslant 0.05$.

\section{RESULTS}

Median and mean of the preoperative antibody levels shown in Table 1 ranked as TF-pAA $<\mathrm{Tn}<a \mathrm{Gal}$. Similar ratio of antibody levels was observed in gastrointestinal cancer patients and donors. The TF-R of different groups demonstrated a significantly better survival: $p<0.05$, hazard ratio (HR) $0.18-0.23$, mean survival time of $\mathrm{R}>\mathrm{WR}$ (Table 2; Fig. $1, a-c$ ). The difference in survival between TF-R and TF-WR in stages I-II and NO as well as in grades G1-2 or tumor volume T2-4 was insignificant. The difference in survival between Tn-R and Tn-WR as well as $\alpha G$ al- $R$ and aGal-WR was insignificant for all patients or their pathomorphological subgroups. A trend to a worse survival of Tn-R and aGal-R (HR 2.43-3.27) in some subgroups was observed (Table 2; Fig. 1, $d$ ).

Table 1. Median and mean of the antibody level in the serum of patients

\begin{tabular}{cccc}
\hline Antibodies & Median & Mean \pm SD, $R$ & Mean \pm SD, WR \\
\hline TF-pAA & 1.255 & $2.999 \pm 2.463$ & $1.134 \pm 0.063$ \\
Tn & 1.805 & $3.913 \pm 2.487$ & $1.380 \pm 0.236$ \\
aGal & 3.790 & $6.293 \pm 2.858$ & $2.180 \pm 0.790$ \\
\hline
\end{tabular}

The specificity of anti-TF-pAA antibodies affinityisolated from cancerous sera was described earlier [18]. In the present study, we analyzed antibodies using IEF and the IgG-Western blotting and showed their polyclonal pattern (Fig. 2).
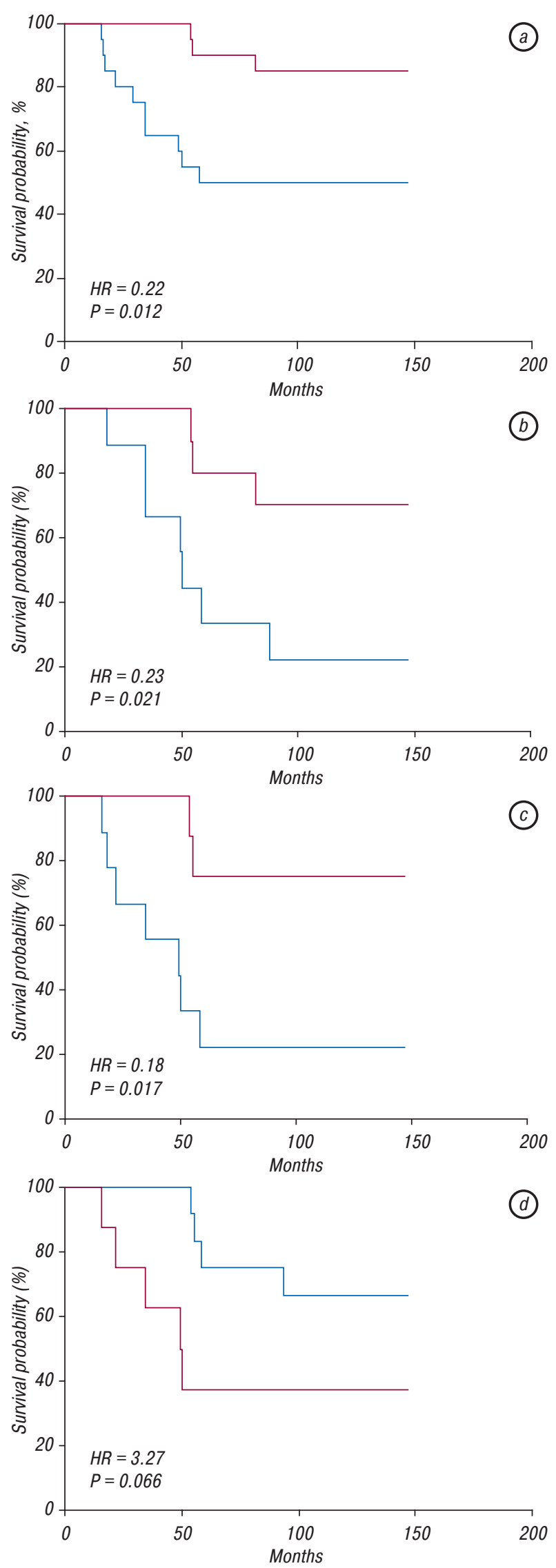

Fig. 1. Probability of survival in $R$ (the level of serum antibodies above or equal to median $\mathrm{M}$, a red line) vs WR (the level below $\mathrm{M}$, a blue line): $a, b, c$ - the relation of the anti-TF-pAA IgG level to survival; $d-$ the relation of the anti- $\alpha$ Gal IgG level to survival. $a$ - all patients; $b$ - patients with an N1-2 status; $c, d$ - patients with G3 tumors 
Table 2. The relation of the antibody level in the serum of patients to survival

\begin{tabular}{|c|c|c|c|c|c|}
\hline $\begin{array}{l}\text { Anti- } \\
\text { bodies }\end{array}$ & Group & $\mathrm{n}$ & $\mathrm{p}$ & HR & $\begin{array}{l}\text { Mean survival time, } \\
\text { months and } 95 \% \mathrm{CL}^{*}\end{array}$ \\
\hline \multirow[t]{10}{*}{ TF-pAA } & All & 56 & 0.012 & 0.22 & $185.8(163.2-208.4)$ \\
\hline & & & & & vs $121.2(84.9-157.4)$ \\
\hline & Stages & 40 & 0.008 & 0.23 & $169.7(136.8-202.6)$ \\
\hline & II-III & & & & vs $95.8(56.7-134.9)$ \\
\hline & T2-4 & 36 & 0.062 & 0.30 & $168.1(130.0-206.2)$ \\
\hline & & & & & vs $100.3(53.3-147.3)$ \\
\hline & N1-2 & 28 & 0.021 & 0.23 & $164.2(123.1-205.3)$ \\
\hline & & & & & vs $81.8(38.1-125.6)$ \\
\hline & G3 & 25 & 0.017 & 0.18 & $167.5(122.1-212.9)$ \\
\hline & & & & & vs $68.7(26.8-110.5)$ \\
\hline \multirow[t]{2}{*}{ Tn } & Stages & 42 & 0.075 & 2.49 & $108.3(66.5-150.1)$ \\
\hline & II-III & & & & vs $157.9(125.2-190.6)$ \\
\hline \multirow[t]{3}{*}{ aGal } & All & 59 & 0.101 & 2.43 & $147.0(115.0-178.9)$ \\
\hline & G3 & 27 & 0.066 & 3.27 & $\begin{array}{c}\text { vs } 181.3(160.8-201.7) \\
98.4(40.4-156.3)\end{array}$ \\
\hline & & & & & vs 159.1 (121.1-197.2) \\
\hline
\end{tabular}

Note: ${ }^{*} \mathrm{R}$ vs WR. $95 \%$ confidence limit $(\mathrm{CL})$ is lower and upper confidence limits shown in brackets. Breast cancer patients that were described in reference [19] are also included in the Table

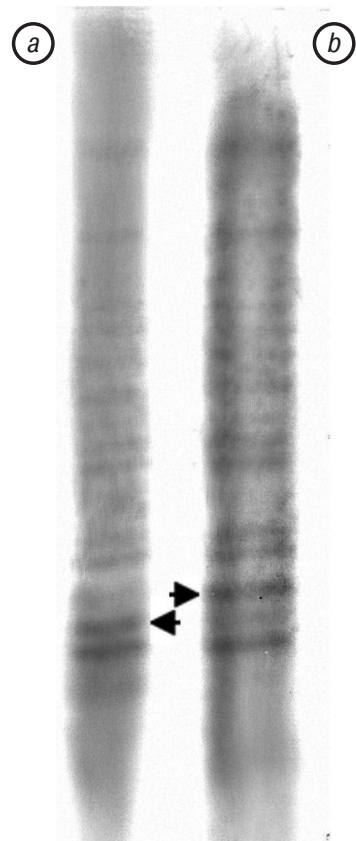

Fig. 2. IEF and the IgG-Western blotting of samples affinityisolated from the serum of cancer patient: $a-$ pAA-reactive antibodies isolated on TFa-spacer-Sepharose; $b$ - pAA-reactive antibodies isolated on TFa-PAA-Sepharose. $a$ and $b$ correspond to samples 2 and 1 that described in [18]. The arrows show the difference in intensity of IgG-bands. The cathode on the top

\section{DISCUSSION}

We demonstrated earlier an association of the level of examined antibodies with survival of patients with gastrointestinal cancer including pathomorphological subgroups. A significantly better survival has been observed in patients with an increased level of antibodies to TF-pAA and Tn-PAA while, on the contrary, a significantly worse survival was observed in an increased antiaGal-PAA IgG level or its postoperative elevation [11]. The present study demonstrates an association of the serum level of anti-TF-pAA IgG and trend to association of anti-Tn and anti-aGal IgG with survival of breast cancer patients. Better survival was revealed in an increased level of anti-TF-pAA antibodies for all group. Similar to patients with gastrointestinal cancer, a better survival of TF-R was observed in subgroups of an ad- vanced breast cancer and G3 tumor (see Table 2). Although a significant differences for Tn-R vs Tn-WR and aGal-R vs WR were not found, values of $H R>2$ and mean survival time for subgroups presented in Table 2 show a trend to worse survival with an increased level of antibodies. The relation of anti-Tn and -aGal antibodies level to survival is less significant in breast cancer than in gastrointestinal cancer. The antibody response to some glycans of enteric microorganisms may be more informative for tumors of digestive tract.

A low level of preoperative anti-TF-pAA IgG dominated in stages III-IV of gastric cancer or in metastases $\mathrm{N} 1-2$, as well as in poorly differentiated carcinoma G3 and it was unfavorable indicator of survival $[11,19]$. Noteworthy, TF-WR in subgroups N1-2 and G3 demonstrated worse survival either in gastrointestinal or in breast cancer. The lower level of anti-aGal IgG has been associated with an advanced breast cancer. A significantly lower anti- $a$ Gal lgG was noted for greater tumor size (T2 + T3 vs T1) and for stage II vs I as well as for low-differentiated breast carcinomas G3 vs G1 + G2 [19]. However, patients with G3 tumor and a lower level of anti-aGal antibodies (aGal-WR) were prone to longer survival (see Table 2).

The low IgG reactivity to TF-pAA in patients with cancer may be due to the presence of serum factors that influence the detection of antibodies. Natural anti-glycan antibodies are mostly lgM-class antibodies. IgM with corresponding specificities can compete with IgG for binding to glycan in ELISA and thereby reduce IgG signal [20]. As a rule, we observed a low IgM reactivity to TF-pAA in serum samples with a high corresponding IgG reactivity but inverse correlation was not revealed. No difference in survival of gastric cancer patients was demonstrated in relation to the anti-TFpAA IgM level [21]. The other serum factor that can reduce $\lg G$ binding to TF-pAA and thereby reduce the signal of antibodies in ELISA is the TF-binding protein galectin 3. Serum levels of galectin 3 in patients with breast and gastrointestinal cancer were significantly elevated especially in metastatic gastrointestinal carcinoma as compared with healthy individuals [22]. A high level of galectin 3 is associated with the worse survival of colorectal cancer patients [23]. The increased level of galectin 3 in advanced cancer and its competition may be an additional factor improving evaluation of survival using determination of IgG reactivity (level) to TF-pAA. This supposition needs further detailed investigations.

Anti-glycan antibodies belong to natural antibodies. Communities of microbiota transmitted from mother to newborn remain stable in lifetime. The natural IgG antibodies are also transferred from mother to fetus and the repertoire of IgG autoantibodies remains stable [20, 24-26]. We observed a similar pattern of anti-TF-pAA, -Tn and - $a$ Gal IgG in sera of grandmother, mother and her adult son; moreover, everyone of donors had a high level of anti-TF-pAA and anti-aGal IgGs. In most gastrointestinal cancer patients the antibodies level is slightly changed but in some patients 
we observed a high level of anti-glycan IgG and its postoperative elevation $[11,12,16]$. A very high level of anti-TF-pAA IgG was marked in eight long-term survivors in stages II-III among 134 patients under study [11]. The twelve-fold change of the anti-TF-pAA IgG level was noted in the follow-up. In one case the stable increase over the background level could be due to prolonged chemotherapy. The anti-TF-pAA IgG level correlated with a count of blood lymphocytes and inversely correlated with neutrophil/lymphocyte ratio [11, 12]. Together these observations point to the adaptive anti-TF-pAA IgG response at least in some patients.

Anti-glycan antibodies associated with survival may be involved in cancer progression through different pathways. Some of them are cross-reactive to histo-blood group related antigens that may be aberrantly expressed in tumors as precursors or incompatible antigens [27]. Some genera of microbiota might express TAG-mimic antigens and stimulate TAG-autoreactive antibodies, which may be beneficial or adverse for cancer patients.

To characterize the specificity of antibodies, antiTF-pAA IgGs were affinity-isolated from the serum samples of long-term survivors, using different TFaconjugated sorbents. Anti-TF-pAA IgG displayed a low specificity to the mucinous fraction isolated from malignant breast tumors except three specimens [28, 29]. Affinity-isolated anti-Tn and - $a$ Gal IgG also were low specific. The cross-reactivity of anti-TF-pAA IgG to different determinants including TFa-unrelated reactivity and reactivity to the pAA carrier without glycans was revealed. We renamed term used earlier "anti-TF IgG" to "anti-TF-pAA IgG". Antibodies were more reactive to TF $\beta$ (Gal $\beta 1-3$ GalNAc $\beta$ ) than to TF (TFa) conjugates, and they were a heterogenic population varying in the cross-reactivity to glycolipidrelated glycans with TF $\beta$ terminal residues, GA1 and $\mathrm{Gb5}_{\text {tri }}[18,28,30]$, the latter being a terminal trisaccharide of Gb5 (stage-specific embryonic antigen 3, SSEA-3), which is expressed in $77.5 \%$ of breast cancer specimens [31]. The specificity to the pAA carrier and 10\%-TFa-pAA but not to the standart 10\%-TFa-PAA, which was inherent in all IgG subpopulations including those isolated without carrier, is enigma [18]. These IgG subpopulations belong to polyclonal antibodies (see Fig. 2). The pAA-mimic (glyco)peptide sequences of unknown proteins may be natural determinants for antibodies. The identification of natural targets could elucidate the direct or indirect relation of a long-lasting anti-TF-pAA IgG immune response to survival.

Dysbiosis is associated with a breast cancer state and severity. The total bacterial DNA load was reduced in the tumor as compared to normal tissue and correlated inversely with an advanced disease [2]. Dysbiosis may influence the tumor development via complex interrelations within the triad: resident microbiota tumor - immune response in which some anti-glycan antibodies might be involved. The dynamic study of the humoral immune response using glycomics together with microbial genomics can elucidate interrelations leading to the carcinogenesis and tumor progression.

\section{CONCLUSIONS}

The present study demonstrates prognostic significance of anti-TF-pAA IgG, which is acceptable not only for gastrointestinal cancer but also for breast cancer. The non-invasive screening test may be a useful supplement in clinical outcome assessment.

\section{ACKNOWLEDGEMENTS}

This study was supported by a grant № 8399 from the Estonian Science Foundation.

\section{REFERENCES}

1. Arrieta MC, Finlay BB. The commensal microbiota drives immune homeostasis. Front Immunol 2012; 3: 33.

2. Xuan C, Shamonki JM, Chung A, et al. Microbial dysbiosis is associated with human breast cancer. PLoS One 2014; 9: e83744.

3. Sheflin AM, Whitney AK, Weir TL. Cancer-promoting effects of microbial dysbiosis. Curr Oncol Rep 2014; 16: 406.

4. Stowell SR, Arthur CM, McBride R, et al. Microbial glycan microarrays define key features of host-microbial interactions. Nat Chem Biol 2014; 1: 470-6.

5. Springer GF, Tegtmeyer H. Origin of anti-ThomsenFriedenreich (T) and Tn agglutinins in man and in white Leghorn chicks. Br J Haematol 1981; 47: 453-60.

6. Ju T, Otto VI, Cummings RD. The Tn antigen-structural simplicity and biological complexity. Angew Chem Int Ed Engl 2011; 50: 1770-91.

7. Galili U, Mandrell RE, Hamadeh RM, et al. Interaction between human natural anti-alpha-galactosyl immunoglobulin $\mathrm{G}$ and bacteria of the human flora. Infect Immun 1988; 56: $1730-7$.

8. Springer GF. Immunoreactive $\mathrm{T}$ and $\mathrm{Tn}$ epitopes in cancer diagnosis, prognosis, and immunotherapy. J Mol Med 1997; 75: 594-602.

9. Campbell BJ, Finnie IA, Hounsell EF, et al. Direct demonstration of increased expression of Thomsen-Friedenreich (TF) antigen in colonic adenocarcinoma and ulcerative colitis mucin and its concealment in normal mucin. J Clin Invest 1995; 95: 571-6.

10. Yu LG. The oncofetal Thomsen-Friedenreich carbohydrate antigen in cancer progression. Glycoconj J 2007; 24: $411-20$

11. Smorodin E, Sergeyev B, Klaamas K, et al. The relation of the level of serum anti-TF, -Tn and -alpha-Gal IgG to survival in gastrointestinal cancer patients. Int J Med Sci 2013; 10: 1674-82.

12. Smorodin EP, Kurtenkov OA, Sergeyev BL, et al. Postoperative change of anti-Thomsen-Friedenreich and Tn IgG level: the follow-up study of gastrointestinal cancer patients. World J Gastroenterol 2008; 14: 4352-8.

13. Smorodin EP, Kurtenkov OA, Sergeyev BL. The level of anti-(GalNAc beta) and anti-para-Forssman disaccharide IgG antibodies in patients with gastrointestinal cancer: relation to survival. Exp Oncol 2013; 35: 89-92.

14. Sobin LH, Gospodarowicz MK, Wittekind C, eds. TNM Classification of Malignant Tumours. $7^{\text {th }}$ Edition. WileyBlackwell, 2009. 336 p.

15. Bovin NV. Polyacrylamide-based neoglycoconjugates as tools in glycobiology. Glycoconj J 1998; 15: 431-46.

16. Smorodin EP, Kurtenkov OA, Sergeyev BL, et al. Antibodies to tumor-associated carbohydrate epitopes in sera of cancer patients and blood donors. Exp Oncol 2001; 23: 109-13.

17. Smorodin EP, Sergeyev BL, Kurtenkov OA. The characterization of IgG antibodies to GalNAc beta-terminated glycans of gastric cancer survivors. Exp Oncol 2014; 36: 38-43. 
18. Smorodin EP, Kurtenkov OA, Sergeyev BL, et al. The characterization of cross-reactive antibodies to ThomsenFriedenreich $\mathrm{a} / \mathrm{b}$ and related glycan-conjugates with polyacrylamide carriers in patients with gastrointestinal cancer. J Clin Cell Immunol 2011; S5.

19. Smorodin EP, Kurtenkov OA, Sergeyev BL, et al. The relation of serum anti-TF, Tn and alpha-Gal IgG antibody levels to cancer progression and histopathological grading. Exp Oncol 2002; 24: 270-3.

20. Muthana SM, Xia L, Campbell CT, et al. Competition between serum $\mathrm{IgG}, \mathrm{IgM}$, and IgA anti-glycan antibodies. PLoS One 2015; 10: e0119298.

21. Kurtenkov O, Klaamas K, Mensdorff-Pouilly S, et al. Humoral immune response to MUC1 and to the ThomsenFriedenreich (TF) glycotope in patients with gastric cancer: Relation to survival. Acta Oncol 2007; 46: 316-23.

22. Iurisci I, Tinari N, Natoli C, et al. Concentrations of galectin-3 in the sera of normal controls and cancer patients. Clin Cancer Res 2000; 6: 1389-93.

23. Barrow H, Rhodes JM, Yu LG. Simultaneous determination of serum galectin-3 and -4 levels detects metastases in colorectal cancer patients. Cell Oncol (Dordr) 2013; 36: 9-13.

24. Hanson LA, Korotkova M, Lundin S, et al. The transfer of immunity from mother to child. Ann NY Acad Sci 2003; 987: 199-206.
25. Peterson CT, Sharma V, Elmén L, et al. Immune homeostasis, dysbiosis and therapeutic modulation of the gut microbiota. Clin Exp Immunol 2015; 179: 363-77.

26. Lacroix-Desmazes S, Mouthon L, Coutinho A, et al. Analysis of the natural human IgG antibody repertoire: lifelong stability of reactivities towards self antigens contrasts with age-dependent diversification of reactivities against bacterial antigens. Eur J Immunol 1995; 25: 2598-604.

27. Hakomori S. Antigen structure and genetic basis of histo-blood groups A, B and O: their changes associated with human cancer. Biochim Biophys Acta 1999; 1473: 247-66.

28. Smorodin EP, Kurtenkov OA, Sergeyev BL, et al. Specificity of human anti-carbohydrate IgG antibodies as probed with polyacrylamide-based glycoconjugates. Glycoconj J 2004; 20: 83-9.

29. Smorodin EP, Kurtenkov OA, Sergeyev BL. The application of human natural polyclonal IgG-antibodies to Thomsen-Friedenreich epitope (TFE) for evaluation of TFE-expression in cancer-associated mucins. Exp Oncol 2000; 22: 44-7.

30. Butschak G, Karsten U. Isolation and characterization of Thomsen-Friedenreich-specific antibodies from human serum. Tumor Biol 2002; 23: 113-22.

31. Chang WW, Lee CH, Lee $\mathrm{P}$, et al. Expression of Globo $\mathrm{H}$ and SSEA 3 in breast cancer stem cells and the involvement of fucosyl transferases 1 and 2 in Globo H synthesis. Proc Natl Acad Sci USA 2008; 105: 11667-72. 